\title{
Von der Vorherrschaft interner Arbeitsmärkte zur dynamischen Koexistenz von Arbeitsmarkt- segmenten
}

Niedriglöhne und nicht-standardisierte Arbeitsverhältnisse haben in den letzten 20 Jahren auf dem ost-westdeutschen Arbeitsmarkt stark zugenommen. Die Fragen nach Reichweite und Grenzen dieses Prozesses sind in Wissenschaft und Politik heftig umstritten und erhalten durch die gegenwärtigen Krisensymptome erneute Aktualität. Der Beitrag vertritt die These einer dynamischen Koexistenz von Teilarbeitsmärkten mit einer Tendenz zur Verbesserung der Arbeitsmarktlage für qualifizierte Arbeit, woraus sich Chancen für abhängig Beschäftigte und Gewerkschaften ergeben können. ${ }^{1}$

\section{Einleitung}

In der zweiten Hälfte des 20. Jahrhunderts gelingt im Rahmen einer für den Kapitalismus einmaligen Prosperitätskonstellation in den frühindustrialisierten Nationen Europas eine Einschränkung des Warencharakters der Arbeitskraft, eine doppelte „De-Kommodifizierung“ (Esping-Andersen; Lutz) des Beschäftigungsverhältnisses. Zum einen binden und schützen große Unternehmen ihre Beschäftigten im Rahmen betriebsinterner Arbeitsmärkte gegen Marktrisiken. Zum anderen wird ein staatlich gestütztes Sicherungsnetz für den Fall des Arbeitsplatzverlustes ausgebaut. Diese Institutionen setzen sich in allen entwickelten kapitalistischen Nationen in der als „Fordismus“ charakterisierten Prosperitätskonstellation durch. Das Normalarbeitsverhältnis und soziale Sicherheit werden zu mehrheitlich geteilten Leitbildern der politischen Kultur (Lutz 2007; Castel/Dörre 2009).

Seit drei Jahrzehnten wird in der Wissenschaft über eine erneute Flexibilisierung und Re-Kommodifizierung des deutschen Arbeitsmarktes gestritten. Viele Autoren diagnostizieren eine weitgehende Vermarktlichung (vgl. Dombois 1999; Beck/Bonß 2001; Davis 2009), während Gegenpositionen eher die Heterogenität und anhaltende Spaltung der Arbeitsmarktstruktur betonen (vgl. Auer/Cazes 2002; Levine et al. 2002; Erlinghagen 2004; Bosch et al. 2007; Keller/ Seifert 2009; Bellmann et al. 2009). Die konträren Großthesen haben eine Welle empirischer Forschung ausgelöst, die zu einer Differenzierung und Relativierung der Umbruchannahmen geführt hat, ohne dass von einer wirklichen Aufklärung des Sachverhalts gesprochen werden kann. Wir führen diesen Umstand auch darauf zurück, dass viele der soziologischen Risikoanalysen auf Arbeitsmarkttheorie verzichten. Aus unserer Sicht sind die in der Tradition der institutionalistischen Ökonomik und Arbeitsmarktsoziologie entwickelten Segmentationsansätze (vgl. Gensior et al. 2004; Michon/Petit 2007; Reich 2008) bzw. Theorien geschlossener und offener Positionssysteme (Sørensen 1983; Kreckel 2004; Groß 2008) dazu geeignet, diese Leerstelle zu schließen.

Segmentationsansätze gehen von einer horizontalen und vertikalen Spaltung des Arbeitsmarktes aus (Übersicht 1; vgl. dazu ausführlich: Köhler/Krause 2010). In der horizontalen Dimension unterscheiden sie interne bzw. geschlossene von externen bzw. offenen Arbeitsmärkten. Als interne Märkte bezeichnen sie Positionssysteme in Erwerbsorganisationen, in denen Mobilität vor allem zwischen Arbeitsplätzen innerhalb desselben Betriebs erfolgt. Interne Arbeitsmärkte sind also durch stabile und langfristige Betriebszugehörigkeiten gekennzeichnet (z.B. Facharbeiter und Ingenieure im Maschinenbau). Externe Märkte haben eine größere Reichweite und erstrecken sich auf ähnliche Positionen in verschiedenen Betrieben, zwischen denen Arbeitskräfte dann mehr oder weniger häufig wechseln (z.B. Baufacharbeiter oder Dozenten in der Weiterbildung). Interne und externe Arbeitsmärkte unterscheiden sich sowohl in ihrem Steuerungsprinzip (Organisation versus Markt) als auch im
Hinblick auf die Arbeitsmarktstrategien von Beschäftigern und Beschäftigten.

In der vertikalen Dimension unterscheiden Segmentationsansätze primäre von sekundären Teilarbeitsmärkten, definiert durch "gute“ bzw. „schlechte“ Erwerbspositionen. Gute Positionen bieten überdurchschnittliche Einkommen und betriebliche Arbeitsplatzsicherheit oder überbetriebliche Beschäftigungssicherheit. Schlechte Positionen sind durch niedrige Einkommen gekennzeichnet und bergen auf externen Arbeitsmärkten zugleich auch ein hohes Beschäftigungsrisiko (Reinigungsbranche, Praktikanten). Die Basis der damit beschriebenen vier Teilarbeitsmärkte stellen geschlossene und offene betriebliche Beschäftigungssysteme dar. Er-

\footnotetext{
Dieser Aufsatz entstand im Rahmen des Forschungsprojektes „Betrieb und Beschäftigung im Wandel. Betriebliche Beschäftigungssysteme und Beschäftigungssicherheit im ost-westdeutschen Vergleich ${ }^{\prime}$, das als Teilprojekt B 2 des Sonderforschungsbereiches (SFB) 580 über "Gesellschaftliche Entwicklungen nach dem Systembruch. Diskontinuitäten, Tradition, Strukturbildung “ von den Universitäten Jena und Halle durchgeführt wird. Wir danken Hajo Holst, Bernd Martens, Gerd Nies und Lothar Wentzel sowie den anonymen Gutachtern der WSI-Mitteilungen für konstruktive Hinweise.
}

Alexandra Krause, Dr., ist wissenschaftliche Mitarbeiterin am Institut für Soziologie der Friedrich-Schiller-Universität in Jena. Arbeitsschwerpunkte: Arbeitsmarktsoziologie und Sozialstrukturforschung. e-mail: alexandra.krause@uni-jena.de Christoph Köhler, Prof. Dr., lehrt am Institut für Soziologie der Universität Jena. Arbeitsschwerpunkte: Arbeitsmarktsoziologie und Sozialstrukturforschung. e-mail: christoph.koehler@uni-jena.de 


\begin{tabular}{|c|c|c|}
\hline \multicolumn{3}{|c|}{ Übersicht 1: Typen und Merkmale von Arbeitsmarktsegmenten } \\
\hline & intern & extern \\
\hline primär & $\begin{array}{l}\text { langfristige Beschäftigung } \\
\text { Arbeitsplatzsicherheit } \\
\text { mittlere und hohe Einkommen }\end{array}$ & $\begin{array}{l}\text { begrenzte Beschäftigungsdauer } \\
\text { Beschäftigungssicherheit } \\
\text { mittlere und hohe Einkommen }\end{array}$ \\
\hline sekundär & $\begin{array}{l}\text { langfristige Beschäftigung } \\
\text { Arbeitsplatzsicherheit } \\
\text { Niedriglöhne }\end{array}$ & $\begin{array}{l}\text { begrenzte Beschäftigungsdauer } \\
\text { Beschäftigungsrisiko } \\
\text { Niedriglöhne }\end{array}$ \\
\hline
\end{tabular}

werbsorganisationen operieren in der Regel mit mehreren, auch unterschiedlichen Beschäftigungssystemen, die ihrerseits an unterschiedliche überbetriebliche Teilarbeitsmärkte angeschlossen sind (Köhler/Krause 2010).

Die Analytik von Segmentationsansätzen legt also eine zweidimensionale Betrachtung nahe, denn die Frage nach der Stabilität von Beschäftigungsverhältnissen ist nicht mit der nach den sozialen Risiken von Arbeitsmarktpositionen zu verwechseln. Stabile Positionen in internen Arbeitsmärkten mit unbefristeten Vollzeitarbeitsverträgen können ebenso wie Jobs auf externen Arbeitsmärkten mit Armutslöhnen einhergehen. Umgekehrt weisen beide Arbeitsmarkttypen Anteile an Positionen mit überdurchschnittlichen Einkommen und Arbeitsplatz- bzw. Beschäftigungssicherheit auf. Arbeitsvertragsformen, die im Zentrum der Forschung zur „Erosion des Normalarbeitsverhältnisses" stehen, stellen nur einen begrenzten Indikator für diese komplexen Zusammenhänge dar.

Diese analytische Perspektive bildet den Hintergrund der Langzeitbeobachtung des deutschen Arbeitsmarktes durch unser Forschungsprojekt (vgl. Fußnote 1; ausführlich: Struck 2006; Köhler et al. 2008; Köhler/Krause 2010; Krause/Köhler 2012), dessen Ergebnisse im Folgenden vorgestellt und diskutiert werden sollen. Das Projekt ist als vergleichende Längsschnittanalyse der ost- und westdeutschen Teilarbeitsmärkte angelegt. Die Entwicklung der horizontalen Segmentation wird über die Stabilität von Beschäftigungsverhältnissen (gemessen anhand von Beschäftigungsdauern) untersucht. Veränderungen der vertikalen Segmentation werden anhand der Größe des Niedriglohnsektors sowie von Arbeitslosigkeits- und Einkommensrisiken nach Betriebsaustritten erfasst. Das Projekt arbeitet mit qualitativen und quantitativen Methoden. ${ }^{2}$

Unser zentraler Befund lässt sich dahingehend zusammenfassen, dass der deutsche Arbeitsmarkt in den letzten zwei Jahrzehnten einen Re-Kommodifizierungsschub durchlaufen hat. Die in den 1950er bis 80er Jahren aufgebaute Vorherrschaft interner Arbeitsmärkte ist gebrochen und einer Struktur der dynamischen Koexistenz und Interaktion der Arbeitsmarktsegmente gewichen. Allerdings gehen wir davon aus, dass sich diese Struktur auf der Basis widerstreitender Interessens- und Machtkonstellationen stabilisieren wird und prognostizieren daher weder eine weitergehende Re-Kommodifizierung in Richtung auf das angelsächsische Modell einer Vorherrschaft externer Märkte noch eine Rückkehr zum alten Modell der Hegemonie interner Arbeitsmärkte. Im Folgenden fassen wir zunächst unsere empirischen Befunde zu Entwicklungstrends zusammen (Abschnitt 2), erörtern sodann unseren neo-institutionalistisch fundierten segmentationstheoretischen Zugang (Abschnitt 3) und ziehen schließlich einige arbeitsmarktpolitische Schlussfolgerungen (Abschnitt 4).

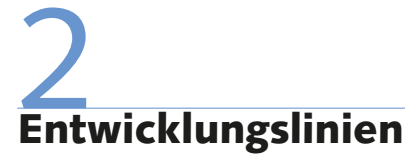

Das Beschäftigungsmodell der alten Bundesrepublik war durch eine Hegemonie interner Arbeitsmärkte mit einem starken öffentlichen Sektor, durch verberuflichte Strukturen, eine starke Institutionalisierung und ein im internationalen Vergleich schwaches sekundäres Segment gekennzeichnet (vgl. Sengenberger 1987; Bosch et al. 2007; Lutz et al. 2007; Streeck 2010). Nimmt man diese Struktur als Referenz für die Entwicklung der Arbeitsmarktsegmentation seit den frühen 1990er Jahren, so zeichnen sich in Ost- und Westdeutschland deutliche Veränderungen $\mathrm{ab}$.

\subsection{HORIZONTALE SEGMENTATION}

In Ostdeutschland hat der Systemwechsel zu höheren Beschäftigungsverlusten als in allen übrigen osteuropäischen Transformationsländern geführt. Die dominanten internen Arbeitsmärkte der Kombinate wurden innerhalb weniger Jahre weitgehend zerstört. Das neu konstituierte Beschäftigungssystem zeichnete sich durch eine massive Externalisierung von Allokationsprozessen aus und näherte sich langsam der westdeutschen Struktur interner und externer Märkte an. So werden etwa in der Industrie und in vielen Branchen des Dienstleistungsgewerbes nur noch Teile der Belegschaften fest beschäftigt, während sich an den Rändern eine starke unfreiwillige und freiwillige Fluktuation entwickelt. Parallel dazu haben externe Teilarbeitsmärkte seit Anfang der 1990er Jahre auch in Westdeutschland an Gewicht gewonnen (Köhler/Loudovici 2008). Auch bei Kontrolle soziodemografischer und konjunktureller Einflussfaktoren nimmt die Beschäftigungsstabilität seit Mitte der 1990er Jahre ab (Grotheer 2008a, b). Innerhalb der ersten Jahre nach Betriebseintritt war die Wahrscheinlichkeit des Wiederaustritts der Beschäftigten aus dem Betrieb in Westdeutschland schon in den 1980er Jahren relativ hoch (Erlinghagen 2004). Allerdings weist Grotheer nach, dass seit den 1990er Jahren auch mittel- und langfristig bestehende Beschäftigungsverhältnisse an Stabilität verloren haben. ${ }^{3}$

Zwar arbeiten Hochschulabsolventen in Ost und West nach wie vor in den stabilsten Beschäftigungsverhältnissen; jedoch verlieren auch die im Laufe der 1990er Jah-

2 Wir nehmen das Postulat der Triangulation ernst: Erstens wurden umfangreiche Analysen der Beschäftigtenstatistik der Bundesagentur für Arbeit (BA) (Grotheer $2008 \mathrm{a}$, b) und der BIBB/ BAuAErwerbstätigenbefragung (Bernhardt et al. 2008) durchgeführt. Zweitens konnte ein auf unsere Fragen spezialisiertes eigenes Betriebspanel mit rund 800 Fällen aufgebaut und von 2002 bis 2010 in vier Wellen erhoben werden (Struck et al. 2007; Schröder et al. 2008; Krause 2011). Drittens haben wir im Laufe der Jahre insgesamt 220 Experteninterviews mit Personalverantwortlichen und Beschäftigten durchgeführt (Köhler/Loudovici 2008; Köhler/Krause 2012).

3 Neuere Befunde zu einer leichten Erhöhung der durchschnittlichen Betriebszugehörigkeitsdauern (Erlinghagen 2010; Rhein 2010) widersprechen unseren Aussagen nicht. Diese im Querschnitt und auf einem hohen Aggregationsniveau erhobenen Daten weisen eine Reihe von Mess- und Interpretationsproblemen auf. Grotheer erfasst im Anschluss an Erlinghagen vollständige Beschäftigungsepisoden und Überlebensraten von Beschäftigungsverhältnissen, die für unsere Fragestellung nach der Ausdehnung externer Arbeitsmärkte den besseren Indikator bilden (Erlinghagen 2004; Grotheer 2008a, S. 70ff.). 
re neu begonnenen akademischen Beschäftigungsverhältnisse an Stabilität. Am instabilsten sind Personen ohne Ausbildung beschäftigt. In Ostdeutschland gewinnen die zu den Erhebungszeitpunkten jeweils bestehenden Beschäftigungsverhältnisse ab Mitte der 1990er Jahre an Stabilität, ohne allerdings das westdeutsche Niveau zu erreichen. Zugleich nimmt die Stabilität neu begonnener Beschäftigungsverhältnisse im Laufe der 1990er Jahre ab, sodass in Ostdeutschland eine stärkere Polarisierung stabiler und instabiler Beschäftigung zu beobachten ist. Wir interpretieren diese Befunde für Ost- und Westdeutschland als Ausdruck der abnehmenden Dominanz interner Arbeitsmärkte in der deutschen Betriebslandschaft. Von einer generellen Externalisierung und Re-Kommodifizierung kann allerdings keine Rede sein, denn die Veränderungen bleiben klar begrenzt.

Durch die Fokussierung auf Vertragsformen und Beschäftigungsdauern werden vor allem von makro-statistischen Analysen endogene Modifikationen von internen Arbeitsmärkten leicht übersehen. Auch bei stabiler Beschäftigung werden hier arbeitsrechtliche und kollektivvertragliche Sicherungen abgebaut, und es kam vor allem in ehemals stark regulierten Großbetrieben zu einer Abkehr von senioritäts- und statusbasierten zugunsten von leistungsbasierten Regeln. Personalauf- und -abbau, aber auch Aufstiege werden mittlerweile stärker an individuelle Leistungen und an die Produktivität und Profitabilität von Betriebsteilen geknüpft. Im Sinne des Subjektivierungsdiskurses findet eine „Vermarktlichung“ interner Arbeitsmärkte statt, ohne dass die Grenzen zwischen ,intern" und „extern" aufgehoben würden. Dieser Trend ist in Ostdeutschland stärker als im Westen vorangeschritten, da die neu konfigurierten internen Arbeitsmärkte von vornherein ohne formelle und informelle Statusrechte angelegt wurden.

\subsection{VERTIKALE SEGMENTATION}

Im Hinblick auf die vertikale Segmentation des Arbeitsmarktes zeigen unsere qualitativen Betriebsanalysen (Köhler/Loudovici 2008), dass vor allem in ausgewählten Bereichen der Einfacharbeit die Betriebs- und Tarifbindung von Beschäftigten aufgelöst wird. So werden auf Arbeitsplätzen in der Produktion, aber vor allem in Serviceabteilungen (Wachdienste, Cafeteria, Reinigung etc.), die ehemals mit Festangestellten be- setzt und tarifvertraglich gesichert waren, Leiharbeiter eingesetzt oder die Arbeiten werden an Fremdfirmen ausgelagert. Sekundäranalysen der Beschäftigtenstatistik der Bundesagentur für Arbeit (BA) (Grotheer 2008a, b) zeigen außerdem, dass die steigende Zahl der Betriebsaustritte zwar häufig mit dem direkten Wechsel in einen neuen Betrieb verbunden ist, heute aber häufiger mit Lohneinbußen einhergehen (besonders ausgeprägt auf dem ostdeutschen Arbeitsmarkt). Die Abstiegsrisiken der Hochschulabsolventen sind nach wie vor am geringsten.

Statistische Analysen der Struktur und Dynamik des Niedriglohnsektors belegen die Reichweite dieses Umbruchs. Westdeutschland lag über Jahrzehnte in internationalen Vergleichen mit Werten um die $15 \%$ Niedriglohnbeschäftigung im Mittelfeld zwischen angelsächsischen Ländern (um $25 \%$ ) und skandinavischen Nationen (um 10 \%). Seit Mitte der 1990er Jahre ist die Niedriglohnquote kontinuierlich auf etwa $20 \%$ angestiegen, wobei Ostdeutschland der Vorreiter ist (Bosch/ Weinkopf 2007).

Schließlich weisen unsere empirischen Analysen auf eine Spaltung zwischen gut qualifizierten und vergleichsweise gut geschützten „Insidern“ auf internen Arbeitsmärkten einerseits und prekär Beschäftigten in offenen betrieblichen Beschäftigungssystemen mit hohen Austauschvolumina an den Rändern der Betriebe andererseits hin. Diese Polarisierung von Stamm- und Randbelegschaften ist in Ostdeutschland stärker ausgeprägt als in Westdeutschland (Grotheer 2008b). Übergänge von offenen in geschlossene Beschäftigungssysteme sind - zumal beim selben Arbeitgeber - in Ostdeutschland seltener (Grotheer 2008b; Schröder et al. 2008).

Wir sehen in der betrieblichen Beschäftigungspolitik in und nach der Krise von 2008/09 einen Hinweis darauf, dass die oben beschriebenen Trends der Expansion externer und sekundärer Arbeitsmärkte vorläufig ausgebremst wurden. In keiner Rezession seit dem Zweiten Weltkrieg war der Rückgang des Bruttoinlandsprodukts (BIP) so groß und der Beschäftigungsrückgang zugleich so schwach wie 2008 und 2009 (Bechmann et al. 2010; Zimmermann/Wey 2010). Von Krise und Personalanpassung war insbesondere das hochproduktive verarbeitende Gewerbe betroffen (Bellmann/
Gerner 2011). In gewisser Weise war hier eine Generalisierung und Vertiefung der in den 1970er Jahren in der Automobilindustrie entwickelten Personalpolitik der „mittleren Linie“ zu erkennen (Köhler/ Sengenberger 1983; Abraham/Houseman 1993; Holst 2010). Die Personalanpassung erfolgte 2008/09 zu einem erheblichen Teil über interne Maßnahmen der Arbeitszeitflexibilisierung, wobei die stark ausgeweitete Kurzarbeit nur einen Teil des Anpassungspotenzials erklärt: Die über mehrere Jahrzehnte hinweg aufgebauten kapazitätsorientierten Arbeitszeitmodelle wurden in der Krise extensiv genutzt (Lehndorff 2010). Beim - gemessen an vorherigen Rezessionen - relativ geringen Personalabbau spielte die Reduzierung atypischer Beschäftigung eine große Rolle, ohne dass auf Entlassungen verzichtet wurde (Hohendanner 2010; Bellmann/ Gerner 2011). Besonders gut erforscht ist der schnelle Abbau und spätere Aufbau der Leiharbeit (Holst 2010). Unsere These ist, dass die sozialstrukturell und ethnisch gemischte atypische Beschäftigung heute das funktionale Äquivalent zu den von Frauen und Migranten dominierten Randbelegschaften der 1970er Jahre bildet.

Bei wichtigen Indikatoren des Sekundarisierungsprozesses am Arbeitsmarkt zeichnet sich über die Krise hinweg eine Stabilisierung ab. Der Anteil des Niedriglohnsektors bleibt auf dem 2006 erreichten hohen Niveau stehen (Kalina/Weinkopf 2010). Die Quote nicht standardisierter Beschäftigung hat die Werte von 2007 im Jahre 2010 wieder erreicht, aber nicht überschritten. Die große Bedeutung der Leiharbeit beim Beschäftigungsaufbau zeigt sich daran, dass ihr Gewicht zuungunsten der geringfügig Beschäftigten zugenommen hat (Statistisches Bundesamt 2011). Die große Frage ist, wie das Beschäftigungssystem mittel- und langfristig auf die im Herbst 2011 prognostizierte tiefe Rezession reagieren wird (siehe Abschnitt 4).

Zusammengefasst zeigt unsere Langzeitbeobachtung, dass das Segment interner Arbeitsmärkte in Westdeutschland seit den 1990er Jahren seine quantitative Dominanz eingebüßt hat. Es entsteht eine Struktur der dynamischen Koexistenz von internen und externen Märkten, die durch den Ausbau des Niedriglohnsektors mit einer Ausweitung des sekundären Arbeitsmarktsegments verbunden ist. Im Osten nähert sich die Arbeitsmarktstruktur nach 
dem Systemwechsel dem westdeutschen Modell im Zeitraffer an und folgt seiner Externalisierungsdynamik. Hierbei entstehen neue Differenzen: In Bezug auf das Gewicht sekundärer Teilarbeitsmärkte haben die ostdeutschen Betriebe den Westen überholt. Dies gilt auch für die De-Institutionalisierung interner Arbeitsmärkte und die Polarisierung zwischen Insidern und Outsidern.

Der Re-Kommodifizierungsschub am ost- und westdeutschen Arbeitsmarkt beinhaltet zwei Schritte hin zu angelsächsischen Beschäftigungsstrukturen mit starken Anteilen externer und sekundärer Arbeitsmärkte. Damit ist aber noch keine Anglosachsifizierung durchgesetzt: Interne Märkte strukturieren in Deutschland nach wie vor in etwa die Hälfte der Beschäftigungsverhältnisse, und ein großer Teil der Betriebslandschaft sperrt sich gegen weitergehende Externalisierungsprozesse (Hohendanner/Gerner 2010; Promberger 2010). Auch sind Rückwärtsbewegungen auf spezifischen Teilarbeitsmärkten und für bestimmte Betriebstypen zu erkennen. Wir gehen deshalb nicht davon aus, dass sich Entwicklungen einer weiterhin voranschreitenden Re-Kommodifizierung auf der einen Seite und die Fortexistenz traditioneller (und insoweit stabiler) Strukturen der Arbeitsmarktspaltung auf der anderen Seite als Alternativen stellen. Vielmehr vermuten wir eine dynamische Koexistenz, in der sich Teilarbeitsmärkte wechselseitig beeinflussen und Grenzverschiebungen in beiden Richtungen möglich sind.

\section{Erklärungsansätze}

Die Analytik von Segmentationsansätzen erlaubt eine mehrdimensionale und differenzierte Beschreibung der sich wandelnden Arbeitsmarktstruktur. Diese Forschungslinie bietet aber auch eine mittlerweile über fünfzigjährige Tradition der Diskussion von Erklärungsansätzen auf der Ebene von Theoremen mittlerer Reichweite (Sesselmeier et al. 2010). So geht der Münchner Segmentationsansatz von hegemonialen Beschäftigerstrategien aus und verbindet diese starke Annahme mit einem sehr offenen sozio-ökonomischen Bezugsrahmen für die Mikro- und Makroebene (Lutz 1987; Sengenberger 1987; vgl. auch Köhler/Krause 2010).

\subsection{IMPULSE DER NEO-INSTITU- TIONALISTISCHEN ÖKONOMIK FÜR DIE MIKROEBENE}

Zur Aktualisierung dieses Ansatzes testen wir das Erklärungsprogramm der neo-institutionalistischen Arbeits- und Personalökonomik (Wolff/Lazear 2001; Sesselmeier et al. 2010), das „Institutionen“ wie das Normalarbeitsverhältnis -ähnlich wie der Münchner Ansatz - aus ökonomischen Strukturen und Beschäftigerinteressen herleitet. Sie geht damit in Erweiterung der Neoklassik von ökonomie-endogenen Mechanismen in der Begründung von Institutionen aus, während die alte institutionalistische Ökonomik diese als exogene Setzung gesellschaftlicher und politischer Kräfte konstruiert.

Zur Modellierung der Beschäftigerinteressen führen wir im Anschluss an die neue Institutionenökonomik (vgl. Martin/ Nienhüser 1998; Baron/Kreps 1999) und arbeits- und industriesoziologische Traditionen (vgl. Hirsch-Kreinsen 2005) betriebliche Personal- und Beschäftigungspolitik ganz allgemein auf zwei grundlegende Bezugsprobleme mit jeweils zwei Varianten zurück: das Personalanpassungsproblem (Verfügbarkeit, Diskontinuität) und das Transformations- oder Leistungsproblem (Kontrolle; Herrschaft). ${ }^{4}$

Das Personalanpassungsproblem betrifft einerseits die Beschaffung und Qualifizierung von Arbeitskräften (Verfügbarkeit) und andererseits den Personalabbau (Diskontinuität). Die Verfügbarkeitshypothese lautet, dass Beschäftiger bei Arbeitskräfteknappheit Bindungsstrategien verfolgen und die Beschäftigten aufgrund ihrer risikoaversen Grundeinstellung darauf eingehen. Die Diskontinuitätshypothese besagt, dass starke Schwankungen im Auftrags- und Arbeitsvolumen sowie die welt- und finanzmarktgetriebene Einengung finanzieller Spielräume die Wahrscheinlichkeit instabiler Beschäftigung erhöhen.

Das Transformations- oder Leistungsproblem formuliert die Annahme, dass Arbeitgeber aufgrund der Unvollständigkeit von Arbeitsverträgen auf die „freiwillige“ Leistungsbereitschaft der Arbeitnehmer angewiesen sind und deshalb auf deren Wunsch nach Arbeitsplatzsicherheit eingehen. Das daraus abgeleitete Kontrollproblem lässt sich wie folgt zusammenfassen: Je höher die Komplexität der Arbeitsaufgabe ist und je weniger die Leistung einzelnen
Personen zugerechnet werden kann, desto schwieriger ist die direkte Kontrolle durch den „Prinzipal“ und desto eher muss der Beschäftiger den Sicherheitserwartungen der Beschäftigten entsprechen (Sesselmeier et al. 2010).

Das Herrschaftsproblem bezieht sich darauf, dass die Durchsetzung von Leistungszielen auch von den Machtpotenzialen und der Legitimität der Personal- und Beschäftigungspolitik gegenüber den Beschäftigten abhängig ist. Von der prinzipiellen Risikoaversion der Beschäftigten ausgehend, folgt daraus die Hypothese, dass die Existenz betrieblicher Interessensvertretungen die Beschäftigungsstabilität im Betrieb erhöht.

Diese Hypothesen lagen unserer Analyse der Struktur und Dynamik der ostwest-deutschen Arbeitsmarktsegmentation zugrunde und sind mittlerweile sowohl qualitativ als auch quantitativ getestet worden (Struck et al. 2007; Köhler et al. 2008; Krause 2011). Insgesamt hat der institutionenökonomische Ansatz eine hohe Erklärungskraft für den Querschnittsvergleich betrieblicher Beschäftigungspolitik bewiesen. Eine Schließung betrieblicher Beschäftigungssysteme ist vor allem dort zu erwarten, wo Anpassungs- und Leistungsprobleme stark ausgeprägt sind. Eine Öffnung betrieblicher Beschäftigungssysteme ist voraussetzungsvoll, weil es weder Verfügbarkeitsprobleme noch Schwierigkeiten bei der Kontrolle der Arbeitsprozesse geben darf. Externe Arbeitsmärkte stellen kein quasi-natürliches Phänomen kapitalistischer Ökonomien dar, denn sie sind sowohl für Beschäftiger als auch Beschäftigte riskant (Krause/Köhler 2012). Dies erklärt dann auch, warum in allen spätmodernen Gesellschaften - unabhängig von ihrer jeweiligen institutionellen Ordnung - ein jeweils erheblicher Teil der Arbeitskräfteallokation über Organisationen und nicht über Märkte erfolgt.

\subsection{ERWEITERUNG DER PERSPEK- TIVEN AUF DER MAKROEBENE}

Um die Dynamik der Arbeitsmarktsegmentation und internationale Unterschiede erklären zu können, müssen wir unseren beschäftigerzentrierten Ansatz allerdings mit Annahmen über sozio-ökonomische

\footnotetext{
4 Ähnliche Ansätze finden sich bei Nienhüser (2009); Sesselmeier (2009); Hohendanner/Gerner (2010).
} 
Entwicklungstendenzen auf der Makroebene verknüpfen. So können wir die Entwicklungstrends der Externalisierung und Sekundarisierung des deutschen Beschäftigungssystems nicht alleine aus der Dynamik und Aggregation personalpolitischer Problemlagen und Strategien herleiten. Die Struktur des Arbeitskräfteangebots ist von übergreifenden organisations- und bevölkerungsdemografischen, sozialstrukturellen bildungspolitischen und kulturellen Prozessen abhängig. Die betrieblichen Handlungsspielräume wiederum sind eng an die Dynamik der globalisierten Finanzund Absatzmärkte gekoppelt. Aus unserer Sicht sind vier Mikro/Makro-Kopplungen von besonderer Bedeutung:

(1) Das betriebliche Personalanpassungsund Diskontinuitätsproblem ist von der Dynamik der Absatz- und Finanzmärkte abhängig. Diese strukturieren die Spielräume für langfristig angelegte Personalstrategien. Sowohl neo-marxistische (Dörre/Holst 2009) als auch neo-institutionalistisch inspirierte Autoren (Streeck 2010) sehen den Globalisierungs- und Finanzialisierungsprozess als massive Schubkraft, die zur nachhaltigen Transformation betrieblicher Beschäftigungspolitik führt. Es besteht kein Zweifel, dass diese Faktoren die Handlungsspielräume der Personalpolitik einschränken und zum Hegemonieverlust interner Arbeitsmärkte in Deutschland beigetragen haben. Wir gehen allerdings davon aus, dass die hiermit verbundenen Kausalitätsannahmen häufig überzogen werden. Zum einen sind vor allem international agierende Unternehmen und ihre Zulieferer betroffen, die nur einen Teil der abhängigen Beschäftigung in Deutschland binden (Bosch et al. 2007; Bellmann/Gerner 2011). Zum anderen hat sich gerade in der Rezession von 2008/09 wieder gezeigt, wie wirkungsvoll die Arbeitszeitflexibilisierung als funktionales Äquivalent zum Personalabbau sein kann. Es gibt also keine direkte Kausalkette von Absatz- und Finanzmärkten hin zu Personalabbaumaßnahmen.

(2) Das betriebliche Verfügbarkeitsproblem variiert direkt mit dem Arbeitskräfteangebot auf den überbetrieblichen Arbeitsmärkten. In der fordistischen Wachstumskonstellation der 1950er bis 70er Jahre haben hohe Wachstumsraten und die damit verbundene Arbeitskräf- teknappheit zum Aufbau der großen internen Arbeitsmärkte und zur praktischen und diskursiven Hegemonie des sogenannten Normalarbeitsverhältnisses beigetragen. Man kann mit Lutz von einer Neutralisierung des Reservearmeemechanismus sprechen (Lutz et al. 2007). Umgekehrt haben der Rückgang der Wachstumsraten und die Zunahme des gesamtwirtschaftlichen Arbeitskräfteangebots in den letzten 25 Jahren durch die geburtenstarken Jahrgänge, die höhere Erwerbsbeteiligung der Frauen und Migrationsprozesse auf vielen Teilarbeitsmärkten verschärfte überzyklische, strukturelle Arbeitskräfteüberschüsse begünstigt, das betriebliche Verfügbarkeitsproblem bei der Beschaffung von Personal reduziert, den Reservemechanismus reaktiviert und damit starke Anreize zur Externalisierung von Allokationsprozessen geschaffen. Mittlerweile zeichnet sich, u.a. bedingt durch die demografische Entwicklung, ein Umschlag in der Arbeitskräfteversorgung für qualifizierte Arbeit ab (vgl. Abschnitt 4).

(3) Die Ausprägung des Verfügbarkeitsproblems wird auch von der sektoralen Struktur von Produktions- und Arbeitssystemen beeinflusst, die Arbeitsplatzstrukturen und damit das Niveau und die Spezifität der Qualifikationsanforderungen bestimmt. Die Besonderheit des deutschen Beschäftigungssystems besteht nach wie vor darin, dass gerade die weltmarktorientierten Sektoren und Betriebe auf komplexe Produktionsprozesse und Produkte, qualifikationsintensive Arbeitsplatzstrukturen und Beruflichkeit (Bosch et al. 2007; Streeck 2010) setzen. Dies setzt auch bei staatlicher und individueller Finanzierung der Aus- und Weiterbildung hohe Humankapitalinvestitionen vonseiten der Betriebe voraus (Marsden 1999; Biehler/ Köhler 2011) und legt Bindungsstrategien und Beschäftigungssysteme vom Typus des internen Marktes nahe. Der oben beschriebene Externalisierungsprozess erklärt sich u.a. daraus, dass in Teilen des wachsenden Dienstleistungssektors atypische Beschäftigung und Instabilität zunehmen, wobei der Staat als Arbeitgeber und - restriktiver - Finanzierer eine große Rolle spielt (Bosch et al. 2007; Hohendanner/Gerner 2010). Im internationalen Vergleich trägt aber die nach wie vor starke industrielle Basis dazu bei, die Reichweite des Externalisierungsprozesses auszubremsen.
(4) Das betriebliche Macht- und Herrschaftsproblem wird direkt durch Verschiebungen im Kräfteverhältnis von $\mathrm{Ka}$ pital und Arbeit beeinflusst. Sowohl das mit der Globalisierung und Finanzialisierung verbundene Drohpotenzial als auch die Reaktivierung des Reservearmeemechanismus auf vielen Teilarbeitsmärkten haben die Gegenmacht der Gewerkschaften auf betrieblicher wie politischer Ebene stark geschwächt. Dieser Verlust an Organisationsmacht geht einher mit einem Verlust an diskursiver Macht. Der noch vor drei Jahrzehnten hegemoniale Diskurs zu sozialer Sicherheit und Umverteilung ist politischmedial konkurrierenden Deutungs- und Legitimationsmustern gewichen, die auch bei Wahlen auf große Akzeptanz stoßen. Diese Machtverschiebungen haben sich in den teilweise weitreichenden Reformen der Arbeitsmarkt- und Sozialpolitik sowie des Arbeitsrechts niedergeschlagen, die u.a. über die Hartz-Gesetzgebung und Förderung atypischer Arbeitsverträge wiederum zur Schwächung der Gegenmacht beigetragen haben. Auf der anderen Seite zeigt die Einstellungsforschung, dass altsozialdemokratische Deutungsmuster und Gerechtigkeitsvorstellungen nach wie vor in der deutschen Bevölkerung dominieren (Krause 2007). Hierin sehen wir einen Begrenzungsmechanismus für weitergehende Externalisierungs- und Sekundarisierungsprozesse (vgl. Promberger 2010).

Die Globalisierung der Absatz- und Finanzmärkte erhöht den Anpassungsdruck bei schwankenden Absatz- und Arbeitsvolumina und bildet damit starke Schubkräfte für eine Veränderung der betrieblichen Beschäftigungspolitik, während die Arbeitskräfteüberschüsse ebenso wie der Abbau an gewerkschaftlicher und politischer Gegenmacht und die damit einhergehenden Sozialstaatsreformen den Personalaustausch erleichtern. Alle drei Faktoren sind in Ostdeutschland stärker ausgeprägt und erklären die Vorreiterfunktion auf dem gesamtdeutschen Arbeitsmarkt, die unsere empirischen Analysen belegen konnten: Über ihre international agierenden Eigentümer und Auftraggeber sind Teile der kleinbetrieblichen und abhängigen ostdeutschen Betriebslandschaft dem Weltmarkt in besonderer Weise ausgesetzt. Die Arbeitskräfteüberschüsse waren in den letzten beiden Jahrzehnten - gemessen am Unterbeschäftigungsvolumen - fast doppelt so hoch wie in Westdeutschland; 
die gewerkschaftliche Gegenmacht ist seit der Wende durchgehend schwächer als in Westdeutschland.

\subsection{REICHWEITE UND GRENZEN DES ANSATZES}

Insgesamt hat sich unsere neo-institutionenökonomische Erklärungsstrategie für die Interpretation der dynamischen Arbeitsmarktsegmentation in Ost- und Westdeutschland bewährt. Hier sind allerdings zwei Einschränkungen zu machen: Erstens geht das Arbeitskräfteangebot, also die Interessen und Orientierungen der Beschäftigten, nur über die stark vereinfachende Annahme der Risikoaversion und daher weitgehend als „black box“ in die Erklärung ein. Damit weist der Ansatz aber eine deutliche Leerstelle auf. Dies gilt auch dann, wenn man - wie wir - am Primat der Nachfrageseite für die Strukturierung von Arbeitsmärkten festhält. Wir können eine derart definierte Beschäftigtenperspektive in unseren bisherigen Erklärungsansatz integrieren, indem wir zunächst das Bezugsproblem der Beschäftigten näher bestimmen, das sie über ihre Beschäftigungsbeziehung zu lösen versuchen: die Sicherung eines kontinuierlichen und existenzsichernden Einkommens. Unter anderem die Berücksichtigung unterschiedlicher Haushaltsregimes, staatlicher Transferleistungen und variierender Mobilitätspräferenzen erlauben dann eine Ausdifferenzierung möglicher Erwerbsstrategien (Köhler/Krause 2010; Krause/Köhler 2012).

Eine zweite Einschränkung liegt darin, dass die historisch gewachsenen institutionellen Ordnungen sowie Machtverhältnisse und Legitimationsmuster für die Entwicklung der Beschäftigungsverhältnisse nachweislich eine große Rolle spielen, bislang allerdings vernachlässigt wurden. Dies ist dann auch das Einfallstor für „kulturalistische“ Vorschläge in der Wirtschafts- und Arbeitsmarktsoziologie, die sowohl in der Tradition des soziologischen Neo-Institutionalismus als auch der neueren Foucault-Interpretation zu suchen sind (vgl. Maurer 2008; Lessenich 2008). Uns erscheint die Notwendigkeit einer derartigen kulturalistischen Wende allerdings nicht plausibel. Zum einen dürfte deutlich geworden sein, dass Verschiebungen in Machtverhältnissen und Diskurswelten in vielfältiger Weise in ökonomische Entwicklungslogiken eingebunden sind. Politische Macht und Institutionen haben im Wirtschaftssystem eine ökonomische Funktion. Zum anderen können kulturelle und politische Dynamiken das ökonomische System überformen, beeinflussen und irritieren, dessen Eigenlogik jedoch nicht auflösen. Wer vom Arbeitsmarkt redet, sollte daher von der Ware Arbeitskraft und Kapitalismus nicht schweigen (vgl. Dörre et al. 2009; Streeck 2010). Soweit man sich - wie wir - auf der Ebene von Theorien mittlerer Reichweite bewegt, sehen wir in den soziologischen Neoinstitutionalismen und verwandten Ansätzen sinnvolle Ergänzungen, nicht aber Alternativen zur neuen Institutionenökonomik.

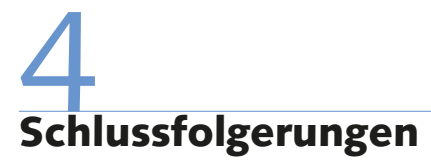

Ergebnis unserer umfangreichen empirischen Analysen ist, dass sich der deutsche Arbeitsmarkt in den letzten 20 Jahren durch einen zwar begrenzten, aber deutlichen Externalisierungs- und Sekundarisierungsschub ausgezeichnet hat. Interne Arbeitsmärkte und das Normalarbeitsverhältnis haben ihre praktische und diskursive Vorherrschaft verloren, und es hat sich eine spannungsgeladene und instabile Koexistenz von internen und externen Arbeitsmärkten entwickelt. Hierbei hat Ostdeutschland eine Vorreiterposition übernommen. Ab 2006 zeichnet sich dann in beiden Landesteilen anhand statistischer Indikatoren eine Ausbremsung des Externalisierungs- und Sekundarisierungsschubs ab, die auch während und nach der Krise von 2008/09 Bestand hat. Als Gesamtbild ergibt sich weder eine weitgehende Re-Kommodifizierung noch eine stabile Spaltung des Arbeitsmarktes. Wir gehen vielmehr von einer neuen Struktur der dynamischen Segmentation aus, in der sich Grenzverschiebungen zwischen internen und externen, aber auch endogene Transformationen innerhalb von Teilarbeitsmärkten vollziehen.

Die Entwicklung des ostdeutschen Beschäftigungssystems lässt sich zusammenfassend als Prozess der doppelten Modernisierung kennzeichnen. Einerseits wurden die Institutionen und Praktiken des „Rheinischen Kapitalismus“ im Sinne einer „einfachen“ Modernisierung übertragen. Andererseits befindet sich eben dieses Kapitalismus- und Beschäftigungsmodell selbst in der Krise, sodass wirkmächtige
Akteure die erweiterten Handlungsspielräume in Ostdeutschland für eine „Modernisierung“ des Modells nutzen. Im Spannungsfeld zwischen den übertragenen westdeutschen Institutionen und den sich neu herausbildenden ökonomischen, sozialen und politischen Strukturen sind die Arbeitsmärkte der Neuen Bundesländer zum Experimentierfeld der Beschäftigungs- und Arbeitsmarktpolitik geworden.

Die Frage ist, ob die aktuell von vielen Autoren für die nahe (und weitere) $\mathrm{Zu}$ kunft prognostizierten Krisentendenzen zu einem weitergehenden Re-Kommodifizierungsschub am deutschen Arbeitsmarkt führen können. Aus der Perspektive unseres Erklärungsmodells gehen wir davon aus, dass in Deutschland ein Übergang zum angelsächsischen Beschäftigungsmodell mit einer Vorherrschaft externer Arbeitsmärkte unwahrscheinlich ist. Wir vermuten vielmehr, dass sich die in den letzten zwei Jahrzehnten herausgebildete neue Struktur der dynamischen Koexistenz von Arbeitsmarktsegmenten stabilisiert. Sollte sich allerdings in den nächsten Jahren eine langanhaltende Strukturkrise des globalen Finanzmarktkapitalismus herausbilden (vgl. Sozialismus, Heft 9/2011), müssten unsere Entwicklungshypothesen umgeschrieben werden.

In dem gegenwärtig von vielen Autoren und von uns vertretenen weniger pessimistischen Szenario einer weltweiten Konjunkturabschwächung und Rezession nehmen die Unsicherheiten auf den internationalisierten Absatz- und Finanzmärkten und damit auch innerhalb der beteiligten Volkswirtschaften auch mittel- und langfristig zu. Damit steigt der Druck auf die Reduktion fixer Personalkosten. Wie die Flexibilitätsforschung seit Jahrzehnten und nicht zuletzt die Beobachtung der Krise von 2008/09 zeigen, führt dieser Druck aber nicht notwendigerweise zur Umstellung von geschlossenen auf offene betriebliche Beschäftigungssysteme mit der Folge einer weiteren Zunahme externer Märkte.

Ein Strategiewechsel auf Externalisierung ist - wie oben gezeigt - auch von der Verfügbarkeit einschlägig qualifizierter Arbeitskraft abhängig. In Bezug auf diese Frage gehen wir mittel- und langfristig von einer sich vertiefenden Spaltung zwischen dem primären und sekundären Arbeitsmarktsegment aus. Im Bereich qualifizierter Arbeit zeichnet sich ein Ende des durch die geburtenstarken Jahrgänge, die zunehmende Frauenbeschäftigung 
und die Wiedervereinigung angetriebenen jahrzehntelangen Angebotswachstums ab. Hintergrund dafür ist die absehbare organisations- und bevölkerungsdemografische Entwicklung: Wir stehen am Anfang eines Prozesses der Verrentung der geburtenstarken Jahrgänge, der auf schrumpfende Nachwuchskohorten trifft. Diese Entwicklung kann in den neuen Bundesländern schon heute beobachtet werden und wird sich Schritt für Schritt auf Gesamtdeutschland ausweiten. Die Arbeitsmarktprognosen des Instituts für Arbeitsmarkt- und Berufsforschung (IAB) zeigen, dass ein Ausgleich über die erhöhte Beschäftigung von Frauen, älteren Arbeitnehmern und Migranten sehr unwahrscheinlich ist.

Damit teilen wir nicht die interessenpolitisch motivierte Dramatisierung und Warnung vor einer Generalisierung der Fachkräfteknappheit und der damit verbundenen Schwächung der „deutschen Wettbewerbsfähigkeit“. Wir gehen aber davon aus, dass sich - in Abhängigkeit von Tiefe und Dauer der prognostizierten Rezession - mittel- und langfristig in vielen Berufen und Teilarbeitsmärkten die Arbeitsmarktposition der Betriebe zugunsten der abhängig Beschäftigten verschlechtern wird. Wenn für die Betriebe Verfügbarkeitsprobleme zunehmen, entwickeln sie Bindungsstrategien und die Beschäftigten können ihre Interessen an Arbeitsplatzsicherheit besser vertreten. Daraus ergeben sich auf vielen Teilarbeitsmärkten Barrieren für weitergehende Externalisierungsprozesse. Die Verbesserung der Arbeitsmarktposition der Beschäftigten wird auch den Handlungsspielraum für die Gewerkschaften erhöhen.

In Bezug auf das sekundäre Arbeitsmarktsegment gehen wir eher von pessimistischen Annahmen aus. Der Ausbau des Niedriglohnsektors begann bereits Mitte der 1990er Jahre, setzte sich über mittler- weile zwei Konjunkturzyklen fort und ist erst seit 2006 zum Stillstand gekommen. Wir vermuten, dass die oben beschriebene mittelfristig mögliche Verbesserung der Arbeitsmarktlage nicht auf das sekundäre Segment durchschlägt, denn erstens ist in den Jahrzehnten der Unterbeschäftigung ein großes gering- oder fehl-qualifiziertes Arbeitskräftepotenzial entstanden, das durch Absolventen ohne Schulabschluss oder Lehre kontinuierlich aufgeladen wird; zweitens stößt dieses Angebot auf einen stagnierenden oder schrumpfenden Sektor der Einfacharbeit; drittens dürften gerade hier Migrationsbewegungen auch im informellen Sektor zur Verschärfung des Reservearmee-Mechanismus beitragen.

Welche politischen Schlussfolgerungen sind daraus zu ziehen? Für die primären externen Teilarbeitsmärkte mit Fachkräftebedarf entstehen mittel- und langfristig neue Chancen für eine offensive Arbeitsmarktpolitik mit dem Ziel der Verbindung von Flexibilität und Sicherheit (Kronauer/Linne 2005; Struck 2006). Dies kann einmal über die Verstärkung aktiver Arbeitsmarktpolitik mit Fortbildung und Umschulung entlang beruflicher Strukturen geschehen, die die nach wie vor beste Verbindung von allokativer Effizienz und Sicherheit auf externen Arbeitsmärkten ermöglichen. Zum anderen wird eine verbesserte Arbeitsmarktlage mehr Austritte aus dem und Eintritte in das Beschäftigungssystem zur Synchronisation von Arbeit und Leben erlauben, die mit Elementen einer Beschäftigungsversicherung gestützt werden könnten (Schmid 2008).

Das seit den 1990er Jahren gewachsene sekundäre Arbeitsmarktsegment mit Niedriglöhnen und hohen Beschäftigungsrisiken ist nicht nur ein moralisches Problem, sondern Gift für den Arbeitsmarkt und die gesamte Gesellschaft. Die arbeitsmarktpolitischen Instrumente einer konsequenten Entprekarisierungspolitik (Dörre) mit einem Ausbau der Grundsicherung, Mindestlöhnen, der Einschränkung nichtstandardisierter Arbeitsverhältnisse usw. sind bekannt; das Problem liegt eher in der politischen Durchsetzung.

Wenn sich an den politischen Konstellationen in Deutschland nichts Grundlegendes ändert, bleibt die starke Spaltung zwischen primären und sekundären Arbeitsmärkten auf absehbare Zeit bestehen. Sie verläuft zwischenbetrieblich aber auch innerhalb von Betrieben und entspricht hier derjenigen von Stamm- und Randbelegschaften. Unter diesen Bedingungen bleibt Gewerkschaften und Betriebsräten der Konflikt zwischen exklusiver und inklusiver Solidarität erhalten. Einerseits besteht ein massives Interesse daran, Substitutionskonkurrenz durch nicht-standardisierte Beschäftigungsverhältnisse einzuschränken (Dörre 2009), andererseits können Randbelegschaften mit unsicherem Status die Stammbelegschaften stabilisieren, sodass Letztere davon profitieren (Detje et al. 2011). In dieser Zwickmühle betreiben viele Interessensvertreter eine Politik der kleinen Schritte, indem sie Übernahmen und Sicherungen einfordern, diese aber immer nur begrenzt durchsetzen können. Unsere Frage ist, ob man nicht von den alten Senioritätssystemen der angelsächsischen Gewerkschaften lernen kann (Köhler/Sengenberger 1983). Hier werden bei Personalabbau Betriebszugehörigkeitsdauern angerechnet und mit Rückrufrechten nach demselben Prinzip verbunden. Über diese Politik wird der belegschaftsinterne Konflikt entschärft: Die betriebsälteren Beschäftigten bleiben gesichert, die betriebsjüngeren haben aber die Chance, über Rückrufrechte Ansprüche auf sichere Arbeitsplätze zu erwerben. Dort, wo Betriebsräte nicht-standardisierte Arbeit und Personalabbau nicht verhindern können, wären die Betroffenen gleichwohl in die Solidargemeinschaft einbezogen. 
Abraham, K. G./Houseman, S. N. (1993): Does Employment Protection inhibit Labor Market Flexibility? Lessons from Germany, France and Belgium, NBER Working Paper (4390), Cambridge, MA

Auer, P./Cazes, S. (2002): Employment stability in an age of flexibility. Evidence from industrialized countries, Geneva

Baron, J. N./Kreps, D. (1999): Strategic Human Resources, New York Bechmann, S./Dahms, V./Fischer, A./Frei, M./Leber, U. (2010): 20 Jahre Deutsche Einheit - Ein Vergleich der west- und ostdeutschen Betriebslandschaft im Krisenjahr 2009. Ergebnisse des IAB-Betriebspanels 2009, IAB-Forschungsbericht (06), Nürnberg

Beck, U./Bonß, W. (2001): Die Modernisierung der Moderne, Frankfurt a. $M$.

Bellmann, L./Fischer, G./Hohendanner, C. (2009): Betriebliche Dynamik und Flexibilität auf dem deutschen Arbeitsmarkt, in: Möller, J./Walwai, U. (Hrsg.): Handbuch Arbeitsmarkt 2009, IAB-Bibliothek (314), Bielefeld, S. 359-401

Bellmann, L./Gerner, H. (2011): Reversed roles? Wage and employment effects of the current crisis, in: Immervoll, H./Peichl, A./Tatsiramos, K. (Hrsg.): Who loses in the downturn? Economic crisis, employment and income distribution, in: Research in Labor Economics 2 (1), S.181-206

Bernhardt, J./Köhler, C./Krause, A. (2008): Sicherheitserwartungen und -konstruktionen im Normalarbeitsverhältnis. Qualitative und quantitative Befunde, in: Köhler, C./Struck, O./Grotheer, M./Krause, A./Krause, I./Schröder, T. (Hrsg.): Offene und geschlossene Beschäftigungssysteme - Determinanten, Risiken und Nebenwirkungen, Wiesbaden, S. 275-306

Biehler, H./Köhler, C. (2011): Berufliche Bildung - Markt oder Staat? Eine kommentierte Literaturübersicht, in: Sonderforschungsbereich (SFB) 580 - Mitteilungen (38), Jena (als download verfügbar)

Bosch, G./Haipeter, T./Latniak, E./Lehndorff, S. (2007): „Demontage oder Revitalisierung? Das deutsche Beschäftigungsmodell im Umbruch“", in: Kölner Zeitschrift für Soziologie und Sozialpsychologie (KZfSS) 59 (2), S. 318-339

Bosch, G./Weinkopf, C. (Hrsg.) (2007): Arbeiten für wenig Geld. Niedriglohnbeschäftigung in Deutschland, Frankfurt a. M.

Castel, R./Dörre, K. (2009): Prekarität, Abstieg, Ausgrenzung - die soziale Frage am Beginn des 21. Jahrhunderts, Frankfurt a. M./New York Davis, G. F. (2009): Managed by the markets: How fincance re-shaped America, Oxford

Detje, R./Menz, W./Nies, S./Sauer, D. (2011): Krise ohne Konflikt? Interessen und Handlungsorientierungen im Betrieb - Die Sicht von Betroffenen, Hamburg

Dörre, K. (2009): Prekarität im Finanzmarktkapitalismus, in: Castel, R./ Dörre, K. (2009): Prekarität, Abstieg, Ausgrenzung - die soziale Frage am Beginn des 21. Jahrhunderts, Frankfurt a. M./New York

Dörre, K./Hajo, H. (2009): Nach dem Shareholder Value? Kapitalmarktorientierte Unternehmenssteuerung in der Krise, in: WSI-Mitteilungen 62 (12), S. 667-674

Dörre, K./Lessenich, S./Rosa, H. (2009): Soziologie - Kapitalismus Kritik, Frankfurt a. $M$.

Dombois, R. (1999): Der schwierige Abschied vom Normalarbeitsverhältnis, in: Aus Politik und Zeitgeschichte (37), S. 13-20

Erlinghagen, M. (2004): Die Restrukturierung des Arbeitsmarktes. Arbeitmarktmobilität und Beschäftigungsstabilität im Zeitverlauf, Wiesbaden
Erlinghagen, M. (2010): Zunehmende Angst vor Jobverlust trotz gleichbleibender Beschäftigungsstabilität, in: Informationsdienst Soziale Indikatoren (44), S. 1-4

Gensior, S./Mendius, H. G./Seifert, H. (Hrsg.) (2004): 25 Jahre SAMF. Perspektiven Sozialwissenschaftlicher Arbeitsmarktforschung, SAMF Arbeitspapier 2004-1, Cottbus

Giesecke, J. (2009): Socio-economic Risks of Atypical Employment Relationships: Evidence from the German Labour Market, in: European Sociological Review 25 (6), S. 629-646

Groß, M. (2008): Klassen, Schichten, Mobilität. Eine Einführung, Wiesbaden

Grotheer, M. (2008a): Beschäftigungsstabilität und -sicherheit in Westdeutschland. Entwicklungsdynamik und Folgen für die soziale Ungleichheit, in: Köhler, C./Struck, O./Grotheer, M./Krause, A./Krause, I./ Schröder, T. (Hrsg.): Offene und geschlossene Beschäftigungssysteme - Determinanten, Risiken und Nebenwirkungen, Wiesbaden S. 65-113 Grotheer, M. (2008b): Beschäftigungsstabilität im Ost-West Vergleich, in: Köhler, C./Struck, O./Grotheer, M./Krause, A./Krause, I./Schröder, T. (Hrsg.): Offene und geschlossene Beschäftigungssysteme - Determinanten, Risiken und Nebenwirkungen, Wiesbaden S. 115-141 Hirsch-Kreinsen, H. (2005): Wirtschafts- und Industriesoziologie, Weinheim/München

Hohendanner, C. (2010): Befristete Arbeitsverträge zwischen Auf- und Abschwung: Unsichere Zeiten, unsichere Verträge? IAB-Kurzbericht (14), Nürnberg

Hohendanner, C./Gerner, H. (2010): Die Übernahme befristet Beschäftigter im Kontext betrieblicher Personalpolitik, in: Soziale Welt 61 (1), S. 27-50 Holst, H. (2010): "Die Flexibilität unbezahlter Zeit" - Die strategische Nutzung von Leiharbeit, in: Arbeit. Zeitschrift für Arbeitsforschung, Arbeitsgestaltung und Arbeitspolitik (2+3), S. 164-177

Kalina, T./Weinkopf, C. (2010): Niedriglohnbeschäftigung 2008, IAQReport 2010-6, Duisburg/Essen

Keller, B./Seifert, H. (Hrsg.) (2009): Atypische Beschäftigungsverhältnisse - Flexibilisierung und soziale Risiken, Berlin

Köhler, C./Junge, K./Schröder, T./Struck, O. (Hrsg.) (2006): Trends in Employment Stability and Labour Market Segmentation. Sonderforschungsbereich (SFB) 580, Mitteilungen (16), Jena

Köhler, C./Krause, A. (2010): Betriebliche Beschäftigungspolitik und Arbeitsmarktsegmentation, in: Böhle, F./Voß, G. G./Wachtler, G. (Hrsg.): Handbuch Arbeitssoziologie, Wiesbaden

Köhler, C./Loudovici, K. (2008): Betriebliche Beschäftigungssysteme und Arbeitsmarktsegmentation, in: Köhler, C./Struck, O./Grotheer, M./ Krause, A./Krause, I./Schröder, T. (Hrsg.): Offene und geschlossene Beschäftigungssysteme - Determinanten, Risiken und Nebenwirkungen, Wiesbaden, S. 31-63

Köhler, C./Sengenberger, W. (1983): Konjunktur und Personalanpassung. Betriebliche Beschäftigungspolitik in der deutschen und amerikanischen Automobilindustrie, Frankfurt a. M./New York

Köhler, C./Struck, O./Grotheer, M./Krause, A./Krause, I./Schröder, T. (Hrsg.) (2008): Offene und geschlossene Beschäftigungssysteme Determinanten, Risiken und Nebenwirkungen, Wiesbaden

Krause, A. (2007): Kündigungsschutzreform und Gerechtigkeit - Einstellungen der Erwerbsbevölkerung, in: WSI-Mitteilungen 60 (5), S. 252-258 Krause, A./Köhler, C. (2012): Arbeit als Ware - Bausteine für eine Theorie flexibler Arbeitsmärkte, Bielefeld (Veröffentlichung voraussichtlich im Mai) 
Krause, I. (2011): Das Verhältnis von Stabilität und Flexibilität auf dem deutschen Arbeitsmarkt, Dissertation Jena 2011 (Veröffentlichung in Vorbereitung)

Kreckel, R. (2004): Politische Soziologie der sozialen Ungleichheit, Frankfurt a. M./New York

Kronauer, M./Linne, G. (Hrsg.) (2005): Flexicurity. Die Suche nach Sicherheit in der Flexibilität, Berlin

Lehndorff, S. (2010): Arbeitszeitpolitik nach der Kurzarbeit, in: Schwitzer, H./Ohl, K./Rohnert, R./Wagner, H. (Hrsg.): Zeit, das wir was drehen! Perspektiven der Arbeitszeit- und Leistungspolitik, Hamburg, S. $39-62$

Lessenich, S. (2008): Die Neuerfindung des Sozialen - Der Sozialstaat im flexiblen Kapitalismus, Bielefeld

Levine, D./Belman, D./Charness, G./Groshen, E./O'Shaughnessy, K. C. (2002): How New is the "New employment Contract"? Upjohn Institute, Kalamazoo

Lutz, B. (1987): Arbeitsmarktstruktur und betriebliche Arbeitskräftestrategie. Eine theoretisch-historische Skizze zur Entstehung betriebszentrierter Arbeitsmarktsegmentation, Frankfurt a. M.

Lutz, B. (2007): Wohlfahrtskapitalismus und die Ausbreitung und Verfestigung interner Arbeitsmärkte nach dem Zweiten Weltkrieg, Forschungsberichte aus dem Zentrum für Höhere Studien (ZHS) 08-1, Halle/Wittenberg

Lutz, B./Köhler, C./Grünert, H./Struck, O. (2007): The German Model of Labour market Segmentation - Tendencies of Change, in: Économies et Sociétés 28 (6), S. 44-65

Marsden, D. (1999): A Theory of Employment Systems: Micro-foundations of Societal diversity, Oxford

Martin, A./Nienhüser, W. (Hrsg.) (1998): Personalpolitik - Wissenschaftliche Erklärung der Personalpraxis, München/Mehring

Maurer, A. (Hrsg.) (2008): Handbuch der Wirtschaftssoziologie, Wiesbaden

Michon, F./Petit, H. (Hrsg.) (2007): Is the concept of labour market segmentation still accurate?, in: Socio-Èconomie du Travail (4) (Sonderheft) Nienhüser, W. (2009): Betriebliche Beschäftigungsstrategien und atypische Arbeitsverhältnisse, in: Keller, B./Seifert, H. (Hrsg.): Atypische Beschäftigungsverhältnisse - Flexibilisierung und soziale Risiken, Berlin, S. $45-65$

Pongratz, H. J./Voß, G. G. (Hrsg.) (2004): Typisch Arbeitskraftunternehmer? Befunde der empirischen Arbeitsforschung, Berlin

Promberger, M. (2010): Leiharbeit im Betrieb: Flexibilität und Prekarität einer atypischen Beschäftigungsform. Habilitationsschrift, Nürnberg/ Erlangen
Reich, M. (Hrsg.) (2008): Segmented Labor Markets and Labor Mobility, Cheltemham/Northhampton

Rhein, T. (2010): Beschäftigungsdynamik im internationalen Vergleich: Ist Europa auf dem Weg zum „Turbo“"-Arbeitsmarkt, IAB-Kurzbericht (1), Nürnberg

Schmid, G. (2008): Entgrenzung der Erwerbsarbeit - Erweiterung der sozialen Sicherheit, in: WSI-Mittelungen 61 (7), S. 358-364

Schröder, T./Struck, O./Wlodarski, C. (2008): „Vordringlichkeit des Befristeten"? Zur Theorie und Empirie offener Beschäftigungssysteme. in: Köhler, C./Struck, O./Grotheer, M./Krause, A./Krause, I./Schröder, T. (Hrsg): Offene und geschlossene Beschäftigungssysteme - Determinanten, Risiken und Nebenwirkungen, Wiesbaden, S. 143-200

Sengenberger, W. (1987): Struktur und Funktionsweise von Arbeitsmärkten. Die Bundesrepublik Deutschland im Vergleich, Frankfurt a. M./New York

Sesselmeier, W. (2009): (De)Stabilisierung der Arbeitsmarktsegmentation? Überlegungen zur Theorie atypischer Beschäftigung, in: Keller, B./ Seifert, H. (Hrsg.): Atypische Beschäftigungsverhältnisse - Flexibilisierung und soziale Risiken, Berlin, S. 67-80

Sesselmeier, W./Funk, L./Waas, B. (2010): Arbeitsmarkttheorien, Würzburg

Sørensen, A. B. (1983): Processes of Allocation to Open and Closed Positions in Social Structure, in: Zeitschrift für Soziologie 12 (3),

S. $203-224$

Statistisches Bundesamt (2011): Beschäftigungszuwachs 2010 zu groBen Teilen von Zeitarbeit getragen, Pressemitteilung Nr.270 vom 19.07., www.destatis.de

Streeck, W. (2010): Reforming Capitalism, Oxford

Struck, O. (2006): Flexibilität und Sicherheit. Empirische Befunde, theoretische Konzepte und institutionelle Gestaltung von Beschäftigungsstabilität, Wiesbaden

Struck, O./Grotheer, M./Schröder, T./Köhler, C. (2007): Instabile Beschäftigung. Neue Ergebnisse zu einer alten Kontroverse, in: Kölner Zeitschrift für Soziologie und Sozialpsychologie (KZfSS) 59 (2), S. 294-317

Wolff, B./Lazear, E. (2001): Einführung in die Personalökonomik, Stuttgart

Zimmermann, K./Wey, C. (Hrsg.) (2010): The Economy, Crises, and the Labor Market. Can Institutions Serve as a Protective Shield for Employment? Applied Economics Quarterly Supplement 61, Berlin 